\title{
BMJ Global Health Teaching patient safety in global health: lessons from the Duke Global Health Patient Safety Fellowship
}

\author{
Bria E Johnston, ${ }^{1}$ Randall Lou-Meda, ${ }^{2}$ Sindy Mendez, ${ }^{2}$ Karen Frush, ${ }^{3}$ Judy Milne,${ }^{4}$ \\ Tamara Fitzgerald, ${ }^{5} \mathrm{~J}$ Bryan Sexton, ${ }^{6}$ Henry Rice ${ }^{1}$
}

\begin{abstract}
To cite: Johnston BE, Lou-Meda R, Mendez S, et al. Teaching patient safety in global health: lessons from the Duke Global Health Patient Safety Fellowship. BMJ Glob Health 2019;4:e001220. doi:10.1136/ bmjgh-2018-001220
\end{abstract}

Handling editor Soumitra Bhuyan

Received 11 October 2018 Revised 27 November 2018 Accepted 19 January 2019

Check for updates

(c) Author(s) (or their employer(s)) 2019. Re-use permitted under CC BY-NC. No commercial re-use. See rights and permissions. Published by BMJ.

${ }^{1}$ Surgery and Global Health, Duke University Medical Center Durham, North Carolina, USA

${ }^{2}$ Pediatric Nephrology Unit, Hospital Roosevelt de Guatemala, Guatemala, Guatemala

${ }^{3}$ Pediatrics, Duke University Medical Center, Durham, North Carolina, USA

${ }^{4}$ Patient Safety Office, Duke University Medical Center, Durham, North Carolina, USA ${ }^{5}$ Pediatric Surgery, Duke University Hospital, Durham North Carolina, USA

${ }^{6}$ Patient Safety Center, Duke University Medical Center Durham, North Carolina, USA

Correspondence to Dr Henry Rice; henry.rice@duke.edu

\section{ABSTRACT}

Health systems in low-income and middle-income countries (LMICs) have a high burden of medical errors and complications, and the training of local experts in patient safety is critical to improve the quality of global healthcare. This analysis explores our experience with the Duke Global Health Patient Safety Fellowship, which is designed to train clinicians from LMICs in patient safety, quality improvement and infection control. This intensive fellowship of 3-4 weeks includes (1) didactic training in patient safety and quality improvement, (2) experiential training in patient safety operations, and (3) mentorship of fellows in their home institution as they lead local safety programmes. We have learnt several lessons from this programme, including the need to contextualise training to local needs and resources, and to focus training on building interdisciplinary patient safety teams. Implementation challenges include a lack of resources and data collection systems, and limited recognition of the role of safety in global health contexts. This report can serve as an operational guide for intensive training in patient safety that is contextualised to global health challenges.

\section{INTRODUCTION}

Patient safety remains a prominent gap in global health systems as patients around the world continue to experience preventable harm, particularly in low-resource settings. ${ }^{1-4}$ More people with conditions amenable to healthcare die due to poor-quality care rather than from non-utilisation of their health system. ${ }^{4}$ Although detailed data on medical errors are limited in low-income and middle-income countries (LMICs), up to two-thirds of all healthcare-related complications occur in LMICs. ${ }^{3}$ The economic impact of medical errors is substantial through lost days of work and higher healthcare costs, further contributing to global poverty. ${ }^{5}$

Patient safety and healthcare quality are increasingly recognised in the global health agenda as a central component of a functional health system. ${ }^{467}$ Concerns about healthcare safety have driven the dissemination of
Summary box

- Although medical errors and complications are a major source of mortality and morbidity around the world, there is a lack of training programmes in patient safety in low-income and middle-income countries (LMICs).

- We confirmed that an intensive fellowship of 3-4 weeks in global health patient safety can successfully teach basic principles of patient safety, quality improvement and infection control to clinicians from LMICs.

- Programmes to train clinicians from LMICs need to be contextualised to local contexts, needs and resources.

- Additional needs to improve patient safety include expansion of resources for training of local staff, enhanced research capacity and training of non-physician staff to build interdisciplinary patient safety teams.

tools to measure and reduce medical errors in LMICs, such as written guidelines, performance improvement strategies, preprocedural checklists and process standardisation programmes..$^{8-11}$ In To Err Is Human, the US National Academy of Medicine (formerly the Institute of Medicine) stresses the need for a strong safety culture as a prerequisite to improving healthcare quality. ${ }^{12}$ Programmes to build a safety culture have been associated with improved clinical outcomes and organisational performance in many high-income countries (HIC) healthcare settings, although their use in LMICs remains limited. ${ }^{13-19}$ However, individual safety programmes at the micro-level (clinic or individual provider) are often not implemented in a sustained fashion in LMICs, with common barriers including a lack of trained personnel, as well as costs, limited data collection systems, and complex cultural, social and political constraints. ${ }^{2489112021}$ 
To overcome the lack of leaders in patient safety in LMICs, programmes to train local healthcare staff in patient safety and quality improvement are required. ${ }^{22-25}$ In this analysis, we present our experience with an intensive fellowship to train patient safety for clinicians from LMICs. Through the training of local leaders in patient safety, the capacity to provide high-quality healthcare can be fundamentally transformed in many areas of global health.

\section{BACKGROUND}

The Duke Global Health Patient Safety Fellowship grew from a collaboration between Duke University and the Roosevelt Hospital/University of San Carlos in Guatemala. Over the recent years, we have expanded this partnership to programmes in healthcare safety, focusing on initiatives to enhance patient safety in Guatemala. ${ }^{26}{ }^{27}$ We conducted a formal needs assessment using semistructured interviews early in this collaboration, which identified several institutional needs to improve patient safety, such as enhanced understanding of the value of measuring medical errors, improved data collection systems and access to tools to modify the safety culture.

In addition to these gaps, we recognised the need for training of local experts in patient safety, such that they could lead long-term, sustained patient safety programmes. In response, we developed the Duke Global Health Patient Safety Fellowship. The goal of this fellowship is to train scholars from LMICs in the practice of patient safety and quality improvement, such that they can lead independent patient safety programmes in their home institution. We have operated three Global Health Patient Safety Fellowship classes to date, with training of seven physicians from Guatemala and Pakistan. Fellows represented several specialties, including surgery, paediatrics, nephrology, anaesthesiology and primary care.

Our fellowship is based on several complementary modes of instruction: (1) didactic training in the theory of patient safety and quality improvement, (2) experiential training in patient safety operations, and (3) mentoring of fellows in their home institution as they lead patient safety and quality improvement initiatives. This report summarises the development of this fellowship, as well as lessons learnt from our early experience which may offer guidance to other patient safety training programmes in global health.

\section{TRAINING PHILOSOPHY}

Our philosophy is derived from the experience in patient safety at Duke University as well as from several national and international patient safety organisations. ${ }^{25}$ Our programme emphasises the value of improving a hospital's organisational functioning as a first step to improving patient safety. Our fundamental belief is that most errors in healthcare are the result of a breakdown in systems rather than the fault of individuals. We seek to achieve a 'Just Culture', which is a learning culture that is constantly improving and is characterised by accountability and error identification. ${ }^{28}$

Our trainees learn core theory and tools of patient safety and quality improvement, such as root cause analysis, as well as failure modes and effects analysis. As effective learning comes from seeing parallel units excel, our trainees share practices by which hospitals across the USA and other LMICs are able to overcome safety challenges. Given the range of challenges across LMICs, our activities follow a modular format that is tailored to each trainee's personal goals as well as institutional resources and needs. The goal is to train experts with the right set of tools to become leaders in their own communities in patient safety. We emphasise safety practices that are guided by implementation science, such that patient safety operations are contextualised to the implementation challenges of each home institution.

We encourage building a strong organisational culture, which is 'an organization's language, behavior, beliefs, values, assumptions, authority, and rituals; all of which define an organization's character and norms'. ${ }^{29-31}$ We teach the value of determinants of organisational readiness, such as change valence and informational assessment. ${ }^{32}$ In doing such, we encourage change management that revolves around three questions-'do we know what it will take to implement this change effectively; do we have the resources to implement this change; and can we implement this change given the situation we currently face?' Although we have not formally assessed how our trainees affect organisational change in their home institutions, future study should include measurements of organisational culture in global health settings.

\section{CURRICULUM DEVELOPMENT}

The fellowship curriculum is structured to provide theoretical knowledge, practical application and need-based adaptation of patient safety and quality improvement practices. We used the WHO's Patient Safety Curriculum Guide as a framework, ${ }^{25}$ and adapted our programme content to emphasise topics in patient safety, quality improvement and hospital operations contextualised to global health challenges (figure 1). Of note, we developed this programme to operate over a time period of 3-4 weeks. Although short-term workshops (programmes of 2-4 days) can provide an introduction to this material, they do not adequately train staff to function as independent patient safety officers. As well, several long-term programmes (1-2 years) exist in patient safety and can serve as a rich resource to build research capacity; these resource-intensive programmes are not accessible for many clinicians from LMICs who lack either the funding or time to devote to this training.

In addition to didactic courses, fellows receive individualised instruction from expert faculty in multiple areas. Content is individualised to each trainee's clinical interests, long-term objectives, institutional needs and gaps in knowledge. Early in each fellowship class, 
Key Content Areas

Teaching Methods

Cultural Adaptation
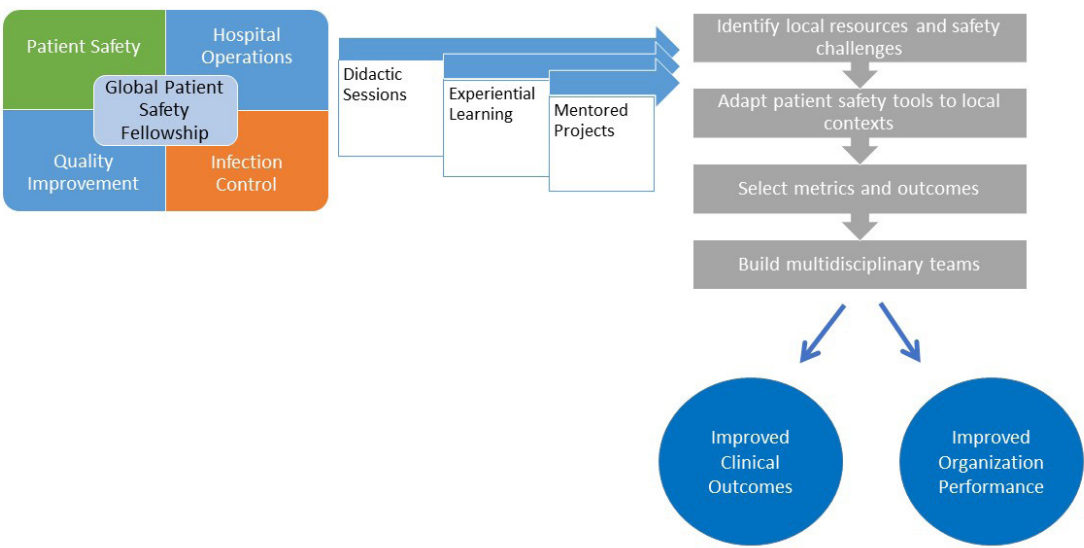

Figure 1 Framework for Duke Global Health Patient Safety Fellowship. Programme content is structured around patient safety, quality improvement, hospital operations and infection control. Teaching is through didactic sessions, experiential learning and mentorship by patient safety experts. The theory of cultural adaptation is emphasised to contextualise content to local resources and safety challenges. The overall goal is to train experts to implement programmes to improve clinical outcomes and organisational performance.

each trainee works with a mentor to develop a personalised curriculum plan to address individual needs. We tailor programme content to each trainee's needs as well as match them with a faculty mentor to facilitate oversight of patient safety initiatives on return to their home institution (table 1). Since participants are selected by their unit or hospital directors, prefellowship partnership between the training and home institutions ensures shared objectives and appropriate adaptation.

\section{DIDACTIC AND EXPERIENTIAL TRAINING}

All fellows participate in several didactic patient safety courses, including the Duke Patient Safety Center Patient Safety Leadership Training and Certification Course, as well as the Team Strategies and Tools to Enhance Performance and Patient Safety course (TeamSTEPPS). The Patient Safety Leadership Training and Certification Course reviews tools, research and frameworks in areas of patient safety, executive rounding, psychological safety, safety culture, resilience and pacing healthcare change. The TeamSTEPPS course uses a 'train-the-trainer' approach to teach evidence-based tools to optimise patient outcomes by improving teamwork skills among healthcare professionals. $^{33}$

Experiential learning is accomplished through observation of hospital quality and patient safety leaders and hospital patient safety operations. Fellows participate in lectures, discussions, mentored simulation exercises and observation of faculty and other Duke safety leadership during regular operations of safety programmes. Following each observation, fellows are debriefed with teaching faculty to discuss content and ways to adapt these programmes to global health settings. During these debriefings, each fellow outlined approaches that incorporate interdisciplinary patient safety teams in their home institutions, and model behaviours to show

Table 1 Topics for Duke Global Health Patient Safety Fellowship

\begin{tabular}{llcl}
\hline Patient safety & Quality improvement & Infection control & Hospital operations \\
\hline Just culture. & Quality improvement. & Framework of infection & Care coordination and \\
Core safety teams. & Process improvement. & control. & handoffs. \\
Safety culture data. & Lean safety principles and & Infection prevention teams. & Institutional safety and \\
Safety culture debriefings. & waste reduction. & Antibiotic control and & operational reports. \\
Global medical errors. & Learning from defects. & stewardship. & Data presentation for \\
Leadership walk-rounds. & Root cause analysis. & Infection control strategies. & executive committees. \\
Safety simulations. & Failure mode and effects & Data collection for surgical & Physician engagement \\
Conflict resolution. & analysis. & site infections. & strategies. \\
Leading as a patient & Proactive risk assessment. & Talking to physicians about \\
safety officer. & Leadership engagement. & & performance. \\
& Quality improvement in & & Fundamentals of physician \\
& complex systems. & & leadership.
\end{tabular}


how professionals of multiple roles can work together to address patient safety challenges. Our fellows learn non-clinical skills to lead interdisciplinary teams, including training in management, leadership, project design and communication. Each of these programmes emphasises how to drive institutional change, manage resources and implement safety innovations. ${ }^{34}$

To complete experiential training, each fellow is required to develop a patient safety project under mentorship of programme faculty. These projects, which are detailed in the following section, are designed such that each fellow can incorporate the theoretical content of our programme as well as lessons from observations of patient safety operations. Although we do not yet know the impact of these experiential projects on clinical outcomes or organisational performance, each fellow is also required to analyse these outcomes in future research projects.

\section{TRAINEE EXPERIENCE AND PROGRAMME OUTCOMES}

We evaluated each trainee's progress and satisfaction using the four-level Kirkpatrick model. ${ }^{35}$ This framework evaluates the success of training through a fourlevel model, measuring each trainee's reaction, learning, behaviour and results. We used a mixed-methods evaluation tool to measure programme success in these areas, with specific attention to reaction, learning and behaviour.

Didactic objectives were assessed by coursework and examination prefellowship and postfellowship, and implementation objectives were assessed by short-term and long-term feedback evaluations from each fellow. Our curriculum evaluation framework was modified to measure success within a global health perspective, such that all participants viewed their training within the context of global health challenges in patient safety as well as content relevant to their representative institutions and home countries.

To evaluate each fellow's reaction, a REDCap survey was distributed to participants via email. As well, we collected data from each trainee's postfellowship summary report. All fellows reported that they felt they could easily implement their newly gained knowledge and skills in their local environment, and strongly agreed they could implement changes in their personal practice and hospitals because of participating in the fellowship.

To assess fellow learning, trainees completed a 33-item multiple-choice assessment before and after training with questions pertaining to patient safety, quality improvement, conceptual analysis and application of patient safety tools. For the most recent fellows $(n=4)$, scores showed an increase after training, with median scores increasing from $56.7 \%$ to $88.8 \% \quad(p=0.003$, Student's t-test).

To evaluate each fellow's behaviour, we used data from the REDCap survey to measure specific examples of how fellows used their newly gained knowledge and skills in their local environment. As our programme is relatively new, specific behaviours were somewhat difficult to measure, although several fellows described new actions that they incorporated into their regular routines, such as leading morning briefings, staff training initiatives or incorporating key performance indicators into their routine reporting systems.

Although the early stages of our fellowship limit our ability to evaluate long-term programme results, most fellows reported an improved ability to lead quality improvement initiatives. Ongoing evaluations will be used to measure changes in institutional practice, organisational performance and patient outcomes as a result of these initiatives. Each recent fellow serves as local patient safety officers, and all are conducting mentored patient safety programmes as well as research initiatives. These initiatives include programmes to improve handwashing compliance, intradepartmental communication, patient safety event reporting and collection of surgery key performance indicators. Three recent fellows are also serving as trainers for other units within their hospital to improve patient safety and quality, and one fellow is educating hospital directors from across Guatemala in patient safety implementation.

\section{ADAPTATION TO GLOBAL HEALTH CHALLENGES}

Adaptation of training to local health contexts is key to effective implementation of global health patient safety programmes. Most existing patient safety programmes are designed for use in HICs, and often require robust data collection platforms, integrated electronic medical records and complex research infrastructure. In contrast, safety programmes in LMICs, as with all frugal technologies, should be developed in collaboration with local leaders to ensure that programmes are responsive to local resources and challenges. ${ }^{36}$ We focused each fellow's training on context-specific learning to ensure that participants learn the correct tools which are applicable to their home institutions infrastructure, resources and needs. In particular, we found it helpful to incorporate examples of safety programmes in US rural and community hospital systems, such as the use of low-resource communication tools, team huddles, morning briefings and skill substitution. ${ }^{37}$

Appropriate cultural adaptation guided by a theoretical framework is essential to guide training in patient safety. We used the ecological validity model to guide our cultural adaptation, which specifies areas of attention when merging interventions across settings, including language, metaphors, content, concept and context. ${ }^{38}$ For example, we recognised early in our fellowship that medical errors were not commonly discussed by staff or leadership in each fellow's home institution. To help frame the importance of reporting medical errors, we identified events from other resource-constrained settings to demonstrate that errors in themselves can be valuable events to understand faults in systems and 


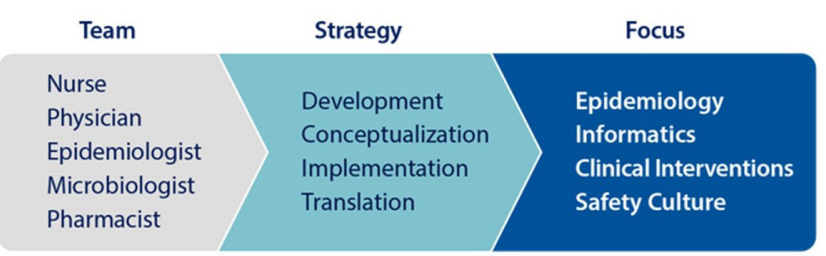

Figure 2 Structure, strategy and focus of interdisciplinary patient safety teams. Our programmes emphasise the value of team structure which comprised staff from different roles. The strategies of team-directed safety programmes can address safety challenges using tools of different fields. Collectively, teams can take on safety projects that are ambitious yet focused, bringing complementary knowledge and approaches. The focus of our programmes emphasises evidence-based patient safety challenges in global health.

improve patient safety. We emphasised the value of viewing medical errors as learning tools rather than for punitive action.

We emphasise the value of interdisciplinary patient safety teams, which comprised staff from different professional roles (figure 2). Team-directed safety programmes have several advantages, particularly within global healthcare settings. Interdisciplinary teams can address complex safety challenges using low-cost tools of different fields. Collectively, teams can take on safety projects that are ambitious yet focused, bringing complementary knowledge and approaches. Teamwork can provide different views to address intersectionality challenges within complex healthcare systems. ${ }^{39}$ Interdisciplinary teams can approach patient safety in a transparent and non-judgemental fashion, using different views to identify positive outcomes from patient safety programmes ('celebrations of success') as well as to identify areas that may require improvement. In low-resource settings, emphasis should be made on identifying easily attainable goals ('low-hanging fruit') to demonstrate to all staff the value of improving patient safety. Although our fellowship classes to date have focused on training of physicians to lead interdisciplinary patient safety teams who can train other team members using a 'train the trainer' approach, our future classes are expanding to include non-physician leaders, including nurses, administrators and non-clinical staff and managers.

\section{IMPLEMENTATION CHALLENGES}

We have learnt several lessons to enhance successful implementation of patient safety efforts in global health settings. For example, fellows reported that there is a general lack of knowledge of safety programmes and appreciation of the value of medical errors in their home institutions, and that most leaders and staff are generally not aware of the magnitude of patient safety problems in their local settings. In response, we now emphasise basic concepts of patient safety, the value of collecting data on medical errors and the advantages of team-based patient safety programmes.

In their postprogramme evaluations, fellows reported several barriers to implementation of patient safety programmes within their own institutions (table 2). The most significant implementation challenges cited by the fellows included a lack of data collection systems to measure medical errors as well as limited institutional resources. Other reported barriers included organisational resistance due to long-standing attitudes and lack of health system knowledge about patient safety. In response, we have helped each fellow gain support of their host institutional leadership in patient safety efforts.

We conducted oral interviews with programme trainees within the first year after completing the programme to assess ongoing implementation challenges of safety programmes in their home institutions. During these interviews, several participants cited early success with patient safety programmes, but also expressed implementation challenges within healthcare systems that are not well versed in the concepts of patient safety. Some of these challenges could be addressed by modifying safety programme choices, such as focusing initially on small projects. For example, one participant stated:

This is a totally new subject. It was shocking to know that this concept has been around the world for more than two decades and we are just getting started. It is a huge challenge to introduce a new concept to our health care system. [Our training] has showed us that we have to start small in making changes...We can then continue to build on that, and by improving our communication and using tools that we have been given, we can improve the environment for both our staff and our patients.

Another fellow expressed: 
[My fellowship training has] helped me teach and educate my staff and coworkers about the importance of patient safety and quality. That together, we need to see the safety culture as a priority and through this we can all be leaders of change in our individual work areas.

\section{CONCLUSION}

Our experience demonstrates that an intensive fellowship of 3-4 weeks can successfully teach basic principles of patient safety and quality improvement that are contextualised to global health challenges. Within a short time period, trainees can be equipped with the core knowledge and set of tools to lead patient safety initiatives at their home institutions. As the central role of patient safety becomes increasingly recognised as essential to functional health systems in LMICs, the training of local patient safety leaders can set the stage to catalyse fundamental improvements in the quality of healthcare. Although validation of the impact of patient safety training on clinical and organisational outcomes is required, the training of local patient safety leaders may contribute to transformational progress in the quality of healthcare around the world.

Contributors BEJ: study design, data collection, data analysis, data interpretation, manuscript writing, manuscript revision. RL-M: study design, data interpretation, manuscript revision. SM: data collection, manuscript writing, manuscript revision. KF: data analysis, manuscript revision. JM: study design, manuscript revision. TF: study design, manuscript revision. JBS: study design, manuscript revision. HR: study design, data collection, data analysis, manuscript revision.

Funding The authors have not declared a specific grant for this research from any funding agency in the public, commercial or not-for-profit sectors.

Competing interests None declared.

Patient consent for publication Not required.

Ethics approval According to the policy activities that constitute research at Duke University this work met criteria for operational improvement activities, and was considered exempt from review by the Duke University Medical CenterCentre Institutional Review Board (Pro00076984)

Provenance and peer review Not commissioned; externally peer reviewed.

Data sharing statement No primary data were used in the development of the paper.

Open access This is an open access article distributed in accordance with the Creative Commons Attribution Non Commercial (CC BY-NC 4.0) license, which permits others to distribute, remix, adapt, build upon this work non-commercially, and license their derivative works on different terms, provided the original work is properly cited, appropriate credit is given, any changes made indicated, and the use is non-commercial. See: http://creativecommons.org/licenses/by-nc/4.0/.

\section{REFERENCES}

1. Ouro-Bang'na Maman AF, Tomta K, Ahouangbévi S, et al. Deaths associated with anaesthesia in Togo, West Africa. Trop Doct 2005;35:220-2.

2. Vivekanantham S, Ravindran RP, Shanmugarajah $\mathrm{K}$, et al. Surgical safety checklists in developing countries. Int J Surg 2014;12:2-6.

3. Jha AK, Larizgoitia I, Audera-Lopez C, et al. The global burden of unsafe medical care: analytic modelling of observational studies. BMJ Qual Saf 2013;22:809-15.

4. Kruk ME, Gage AD, Arsenault C, et al. High-quality health systems in the sustainable development goals era: time for a revolution. Lancet Glob Health 2018;6:1252.

5. Peters DH, Garg A, Bloom G, et al. Poverty and access to health care in developing countries. Ann N Y Acad Sci 2008;1136:161-71.
6. Makary MA, Sexton JB, Freischlag JA, et al. Operating room teamwork among physicians and nurses: teamwork in the eye of the beholder. J Am Coll Surg 2006;202:746-52.

7. Raju TN, Kecskes S, Thornton JP, et al. Medication errors in neonatal and paediatric intensive-care units. Lancet 1989;2:374-6.

8. Haynes AB, Weiser TG, Berry WR, et al. A surgical safety checklist to reduce morbidity and mortality in a global population. $N$ Engl J Med 2009;360:491-9.

9. Perry WRG, Bagheri Nejad S, Tuomisto K, et al. Implementing the WHO safe childbirth checklist: lessons from a global Collaboration. BMJ Glob Health 2017;2.

10. Leotsakos A, Zheng $\mathrm{H}$, Croteau $\mathrm{R}$, et al. Standardization in patient safety: the WHO high 5S project. Int J Qual Health Care 2014;26:109-16.

11. Rowe AK, de Savigny D, Lanata CF, et al. How can we achieve and maintain high-quality performance of health workers in low-resource settings? The Lancet 2005;366:1026-35.

12. Kohn LT, Corrigan JM, Donaldson MS. To err is human: building a safer health system. Washington DC: National Academies Press, 2000.

13. Fan CJ, Pawlik TM, Daniels T, et al. Association of safety culture with surgical site infection outcomes. J Am Coll Surg 2016;222:122-8.

14. Profit J, Lee HC, Sharek PJ, et al. Comparing NICU teamwork and safety climate across two commonly used survey instruments. BMJ Qual Saf 2016;25.

15. Sexton JB, Berenholtz SM, Goeschel CA, et al. Assessing and improving safety climate in a large cohort of intensive care units. Crit Care Med 2011;39:934-9.

16. Sexton JB, Makary MA, Tersigni AR, et al. Teamwork in the operating room: frontline perspectives among hospitals and operating room personnel. Anesthesiology 2006;105:877-84.

17. Zimmermann N, Küng K, Sereika SM, et al. Assessing the Safety Attitudes Questionnaire (SAQ), German language version in Swiss university hospitals--a validation study. BMC Health Serv Res 2013;13.

18. Joshi S, Diwan V, Joshi R, et al. "How Can the Patients Remain Safe, If We Are Not Safe and Protected from the Infections"? A Qualitative Exploration among Health-Care Workers about Challenges of Maintaining Hospital Cleanliness in a Resource Limited Tertiary Setting in Rural India. Int J Environ Res Public Health 2018;15.

19. Smiley K, Ofori L, Spangler C, et al. Safety culture and perioperative quality at the Volta river authority hospital in Akosombo, Ghana. World J Surg 2019;43:23.

20. Peiris D, Thompson SR, Beratarrechea A, et al. Behaviour change strategies for reducing blood pressure-related disease burden: findings from a global implementation research programme. Implementation Sci 2015;10:1-15.

21. Hirschhorn LR, Ramaswamy R, Devnani M, et al. Research versus practice in quality improvement? understanding how we can bridge the gap. Int J Qual Health Care 2018;30(suppl_1):24-8.

22. Ginsburg LR, Dhingra-Kumar N, Donaldson LJ. What stage are low-income and middle-income countries (LMICs) at with patient safety curriculum implementation and what are the barriers to implementation? a two-stage cross-sectional study. BMJ Open 2017;7:e016110.

23. Huang GC, Newman LR, Tess AV, et al. Teaching patient safety: Conference proceedings and consensus statements of the millennium conference 2009. Teach Learn Med 2011;23:172-8.

24. Leotsakos A, Ardolino A, Cheung R, et al. Educating future leaders in patient safety. J Multidiscip Healthc 2014;7:381-8.

25. World Health Organization. Who multi-professional patient safety curriculum guide. Geneva: World Health Organization, 2011.

26. Rice HE, Lou-Meda R, Saxton AT, et al. Building a safety culture in global health: lessons from Guatemala. BMJ Glob Health 2018;3.

27. Taicher BM, Tew S, Figueroa L, et al. Implementation of a colourcoded universal protocol safety initiative in Guatemala. BMJ Qual Saf 2018;27:593-9.

28. Boysen PG. Just culture: a foundation for balanced accountability and patient safety. Ochsner J 2013;13:400-6.

29. Scott T, Mannion R, Davies H, et al. The quantitative measurement of organizational culture in health care: a review of the available instruments. Health Serv Res 2003;38:923-45.

30. Kralewski JE, Wallace W, Wingert TD, Knutson DJ, et al. The effects of medical group practice organizational factors on physicians' use of resources. J Healthc Manag 1999;44:167-84. discussion 82-3.

31. Kaissi A, Kralewski J, Curoe A, et al. How does the culture of medical group practices influence the types of programs used to assure quality of care? Health Care Manage Rev 2004;29:129-38.

32. Weiner BJ. A theory of organizational readiness for change. Implementation Sci 2009;4. 
33. Agency for Healthcare Research and Quality. TeamSTEPPS 2.0. Rockville, MD: Agency for Healthcare Research and Quality, 2018.

34. Grumbach K, Bainbridge E, Bodenheimer T. Facilitating improvement in primary care: the promise of practice coaching. Issue Brief 2012;15:1-14.

35. Kirkpatrick D. Great ideas revisited. Techniques for evaluating training programs. Revisiting Kirkpatrick's four-level model. Training and Development 1996;50:54-9.

36. Howitt P, Darzi A, Yang G-Z, et al. Technologies for global health. The Lancet 2012;380:507-35.
37. Frush $\mathrm{K}$, Chamness $\mathrm{C}$, Olson $\mathrm{B}$, et al. National quality program achieves improvements in safety culture and reduction in preventable harms in community hospitals. Jt Comm J Qual Patient Saf 2018:44:389-400.

38. Bernal G, Bonilla J, Bellido C. Ecological validity and cultural sensitivity for outcome research: issues for the cultural adaptation and development of psychosocial treatments with Hispanics. $J$ Abnorm Child Psychol 1995;23:67-82.

39. Larson E, George A, Morgan R, et al. 10 best resources on... intersectionality with an emphasis on low- and middle-income countries. Health Policy Plan 2016;31:964-9. 